\title{
Multiple target three-dimensional coordinate estimation for bistatic MIMO radar with uniform linear receive array
}

\author{
Jun Li ${ }^{1 *}$, Huan Li ${ }^{1}$, Libing Long ${ }^{1}$, Guisheng Liao ${ }^{1}$ and Hugh Griffiths ${ }^{2}$
}

\begin{abstract}
A novel scheme to achieve three-dimensional (3D) target location in bistatic radar systems is evaluated. The proposed scheme develops the additional information of the bistatic radar, that is the transmit angles, to estimate the 3D coordinates of the targets by using multiple-input multiple-output techniques with a uniform circular array on transmit and a uniform linear array on receive. The transmit azimuth, transmit elevation angles and receive cone angle of the targets are first extracted from the receive data and the 3D coordinates are then calculated on the basis of these angles. The geometric dilution of precision which is based on the root Cramer-Rao bound of the angles, is derived to evaluate the performance bound of the proposed scheme. Further, an ESPRIT based algorithm is developed to estimate the 3D coordinates of the targets. The advantages of this scheme are that the hardware of the receive array is reduced and the 3D coordinates of the targets can be estimated in the absence of the range information in bistatic radar. Simulations and analysis show that the proposed scheme has potential to achieve good performance with low-frequency radar.
\end{abstract}

Keywords: Bistatic, MIMO radar, 3D coordinates, Circular array, GDOP

\section{Introduction}

Multiple-input multiple-output (MIMO) radar is a relatively new term in the radar field, inspired by the MIMO technique in communications. MIMO radar has multiple transmit channels and multiple receive channels, and the transmit channels can be separated by waveforms, or time, or frequencies, or polarizations at each receiver. So the number of channels of a MIMO radar is increased substantially compared to its single-input multipleoutput counterparts. Most of the advantages of the MIMO radar come from increasing the number of channels. Two main classes of MIMO radar have been proposed: with widely separated antennas [1] and with co-located antennas [2]. The first class utilizes the different scattering properties of a target from sufficiently spaced antennas to improve the performance of the systems. The second class allows the improvement of the

\footnotetext{
* Correspondence: junli01@mail.xidian.edu.cn

${ }^{1}$ National Lab of Radar Signal Processing, Xidian University, Xi'an 710071, China

Full list of author information is available at the end of the article
}

radar performances by coherent processing the multiple channels.

A scheme of bistatic MIMO radar has recently been proposed for target localization [3]. Bistatic MIMO radar has the potential advantages both of bistatic radar, such as reduced space loss, covert operation, and reduced susceptibility to jamming [4], and of MIMO radar, such as high spatial resolution and additional spatial degrees of freedom [2]. Also, bistatic MIMO radar has the particular advantage of being able to obtain the target angles with respect to the transmit array (direction of departure) by processing the received data [3]. Several publications have studied direction of departure and direction of arrival estimation for bistatic MIMO radar [5-9]. Multiple target localization without range information can be achieved by using the estimated angles. However, only two-dimensional (2D) Cartesian coordinates can be obtained from the estimated 2D angles. In [10], both the transmit and the receive array are configured as uniform circular arrays UCAs. A trilinear decomposition-based algorithm is developed to estimate the four-dimensional angles of the targets in bistatic 
MIMO radar. In fact, 3D angles are sufficient to locate the targets. It is well known that the localization performance of the bistatic radar is related to the location of the targets. However, to the best of the authors' knowledge, there is no published work on evaluating the localization performance of bistatic MIMO radar in a Cartesian coordinate system.

In this article, a bistatic MIMO radar system with transmit UCA and receive uniform linear array (ULA) is constructed. The 3D coordinates of the targets are then obtained by estimating the transmit azimuth angle, transmit elevation angle and receive cone angle from receive data. The geometric dilution of precision (GDOP) of the system is developed based on the Cramer-Rao bound (CRB) of the angles estimation. The ESPRIT algorithm with phase mode excitation is derived to estimate the $3 \mathrm{D}$ angles. The range of the target is not required in target 3D coordinates estimation and the time synchronization constraint of the bistatic radar is relaxed.

The reminder of the article is organized as follows. The bistatic MIMO radar system model is introduced in Section 2. In Section 3, the GDOP bound is developed to evaluate the performance potential of the proposed scheme. An ESPRIT-like algorithm is developed to estimate the 3D coordinates of the targets in Section 4. The proposed scheme is tested via simulations and analysis, which appear in Section 5. Finally, Section 6 concludes the article.

\section{Bistatic MIMO radar system model}

Figure 1 shows the geometry of the bistatic MIMO radar. The transmit array is a UCA with $N$ elements and the receive array is a ULA with $K$ elements. The element spacing of the linear array is equal to a half-wavelength. We take the location of the transmitter center $O$ as the origin of the coordinate system. The $x-y$ plane is on the ground plane and the $z$-axis points vertically upwards. For simplicity and without loss of generality, we put the receive ULA along the $y$-axis. $A$ is the reference point of the receiver and the length of the baseline $O A$ is $L_{b}$. The elements of the transmitter are uniformly distributed over the circumference of a circle of radius $r$ in the $x-y$ plane. The spacings of both transmit and receive array elements are a half-wavelength. $\alpha \in[0, \pi], \theta \in\left[0, \frac{\pi}{2}\right]$ and $\varphi$ $\in\left[-\frac{\pi}{2}, \frac{\pi}{2}\right]$ are the receive cone angle transmit elevation angle and transmit azimuth angle respectively.

Here, we assume that the transmitted waveforms $\left\{s_{n}(t)\right\}_{n=1}^{N}$ are orthogonal to each other, that is

$$
\int s_{m}(t) s_{k}^{*}(t-\tau) d \tau=\delta_{m k}
$$

where $(g)^{*}$ denotes the conjugate operator.

Assume that $P$ targets with the same range are distributed over different angles. The received signal at the $l$ th pulse period can be expressed as follows:

$$
\mathbf{x}_{l}(t)=\sum_{i=1}^{P}\left[\rho_{i} \mathbf{a}\left(\alpha_{i}\right) \mathbf{b}^{T}\left(\theta_{i}, \varphi_{i}\right) \mathbf{s}(t) e^{j 2 \pi f_{D i} l}\right]+\mathbf{w}_{l},
$$

where $\rho_{i}$ is the signal-reflected coefficient of the $i$ th target. $\left(\alpha_{i}, \theta_{i}, \varphi_{i}\right)$ denotes the corresponding angle of the $i$ th target and $f_{D i}$ denotes the Doppler frequency of the $i$-th target. $\mathbf{w}_{l}$ is Gaussian white noise with covariance $\sigma^{2}$.

$$
\begin{aligned}
& \mathbf{s}(t)= {\left[s_{1}(t), s_{2}(t) \ldots s_{N}(t)\right]^{T}, } \\
& \mathbf{a}(\alpha)= {\left[1, e^{j \pi \cos \alpha}, \ldots e^{j(K-1) \pi \cos \alpha}\right]^{T}, } \\
& \mathbf{b}(\varphi, \theta)=\left[e^{j 2 \pi r \sin \theta \cos \left(\varphi-\gamma_{0}\right) / \lambda}, e^{j 2 \pi r \sin \theta \cos \left(\varphi-\gamma_{1}\right) / \lambda}, \ldots\right. \\
&\left.e^{j 2 \pi r \sin \theta \cos \left(\varphi-\gamma_{N-1}\right) / \lambda}\right]^{T},
\end{aligned}
$$

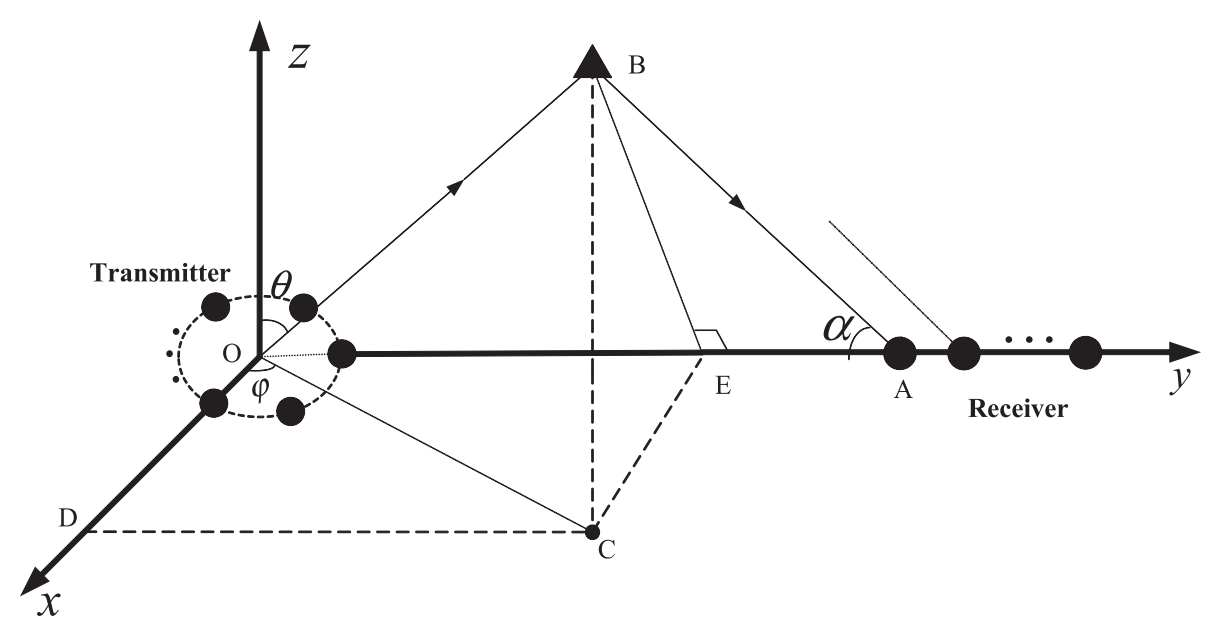

Figure 1 Geometry of the bistatic MIMO radar. 
where $\gamma_{n}=2 \pi n / N, n=0, \ldots, N-1$. The channel separation of the MIMO radar can be achieved by a bank of matched filters in the receiver [3]. The result at the $l$ th pulse period is as follows:

$$
\mathbf{X}(l)=\sum_{i=1}^{P} \rho_{i} \mathbf{a}\left(\alpha_{i}\right) \mathbf{b}^{T}\left(\theta_{i}, \varphi_{i}\right) e^{j 2 \pi f_{D i} l}+\mathbf{W}_{l}
$$

Stacking the matrix $\mathbf{X}(l)$ as a vector, Equation (6) can be written in the form of the $K N \times 1$ vector:

$$
\mathbf{x}_{l}=\mathbf{A h}+\mathbf{n}_{l}
$$

where $\mathbf{A}=\left[\mathbf{a}\left(\alpha_{1}\right) \otimes \mathbf{b}\left(\varphi_{1}, \theta_{1}\right), \mathbf{a}\left(\alpha_{2}\right) \otimes \mathbf{b}\left(\varphi_{2}, \theta_{2}\right), \ldots, \mathbf{a}\left(\alpha_{P}\right) \otimes\right.$ $\left.\mathbf{b}\left(\varphi_{P} \theta_{P}\right)\right]$ and $\otimes$ denotes the Kronecker product. $\mathbf{h}_{l}=$ $\left[\rho_{1} e^{j 2 \pi f_{D 1} l}, \rho_{2} e^{j 2 \pi f_{D 2} l}, \ldots . \rho_{P} e^{i 2 \pi f_{D P} l}\right]^{T}$.

For $L$ pulses, the signal model can be expressed as

$$
\mathbf{Y}=\mathbf{A H}+\mathbf{W}
$$

where $\mathbf{Y}=\left[\mathbf{x}_{1}, \ldots, \mathbf{x}_{L}\right]$ with the size of $K N \times L . \mathbf{H}=\left[\mathbf{h}_{1}\right.$, $\left.\mathbf{h}_{2}, \ldots, \mathbf{h}_{L}\right]$ and $\mathbf{W}=\left[\mathbf{n}_{1}, \ldots, \mathbf{n}_{L}\right]$. It has been proven that the matrix $\mathbf{W}$ has the same statistical properties as the receive noise $\mathbf{w}_{l}$ in the case of orthogonal transmit waveforms [11].

\section{Performance bound of the estimation 3.1. CRB of the 3D angles}

The CRB provides a lower bound of the mean square error of the angle estimation by any unbiased estimator. Following the approach in [12], the CRB for 3D angles of multiple targets is calculated here to obtain the bound of angle estimation of the proposed scheme. The Fisher information matrix (FIM) for the angles can be calculated as follows:

$$
\mathbf{F}(\boldsymbol{\xi})=\left[\begin{array}{lll}
\mathbf{F}_{1,1} & \mathbf{F}_{1,2} & \mathbf{F}_{1,3} \\
\mathbf{F}_{2,1} & \mathbf{F}_{2,2} & \mathbf{F}_{2,3} \\
\mathbf{F}_{3,1} & \mathbf{F}_{3,2} & \mathbf{F}_{3,3}
\end{array}\right]
$$

where $\boldsymbol{\xi}=[\boldsymbol{\alpha}, \boldsymbol{\varphi}, \boldsymbol{\theta}] . \quad \boldsymbol{\alpha}=\left[\begin{array}{llll}\alpha_{1} & \alpha_{2} & \cdots & \alpha_{P}\end{array}\right], \quad \boldsymbol{\varphi}=$ $\left[\begin{array}{llll}\varphi_{1} & \varphi_{2} & \cdots & \varphi_{P}\end{array}\right]$ and $\boldsymbol{\theta}=\left[\begin{array}{llll}\theta_{1} & \theta_{2} & \cdots & \theta_{P}\end{array}\right]$. The derivation of the submatrices of the FIM can be found in Appendix 1. The CRB of the 3D angles of the targets can be obtained by inverting the FIM

$$
\mathbf{C}(\boldsymbol{\xi})=\operatorname{diag}\left(\mathbf{F}^{-1}(\boldsymbol{\xi})\right)
$$

where $\operatorname{diag}(g)$ denotes a vector constructed by the diagonal elements of matrix.

\subsection{GDOP}

As the localization performance of bistatic radar depends on the location of the target, we will analyze the estimate error of the 3D coordinates of the targets in different location. The uncertainties of the measured angles will propagate to the coordinate values according to the error propagation equation [13] as follows:

$$
\Delta \mathbf{e}=\mathbf{T}^{-1} \Delta \mathrm{v}
$$

where $\Delta \mathbf{e}=[\Delta x, \Delta y, \Delta z]^{T}$ is the error of the coordinates. $\Delta \mathbf{v}=[\Delta \alpha, \Delta \theta, \Delta \varphi]^{T}$ is the error of the estimated angles. $\mathbf{T}$ is the error propagation matrix which is derived in Appendix 2. The performance bound of the 3D coordinates estimation can be obtained from (11) by using the root Cramer-Rao bound (RCRB) as the error of the estimated angles, that is

$$
\Delta \overline{\mathrm{e}}=\mathbf{T}^{-1} \sqrt{\mathbf{C}}
$$

where $\Delta \overline{\mathrm{e}}=[\Delta \bar{x}, \Delta \bar{y}, \Delta \bar{z}]^{T}$. The GDOP metric for 3D coordinates is defined as follows:

$$
\mathrm{GDOP}=\sqrt{\Delta \bar{x}^{2}+\Delta \bar{y}^{2}+\Delta \bar{z}^{2}}
$$

In fact, Equation (13) described the bound of the root mean square error (RMSE) of the 3D coordinate estimation of the target at difference location.

\subsection{Analysis of the performance bound}

The GDOPs of the proposed scheme are plotted in Figure 2. The GDOPs in the plane of $z=35 \mathrm{~km}$ are plotted according to (12) and (13). $T$ and $R$ in the Figure indicate the locations of transmitter and receiver respectively. $T$ is located at the origin of the coordinates system and $R$ is located at $[0,100 \mathrm{~km}, 0]$. The Figure shows the performance bound of the proposed scheme. It can be observed that the performance of 3D coordinates estimation varies with the range between the target and the transmitter. It can be seen in Figure 2 that the estimation error can reach several meters when the range of the target is within $50 \mathrm{~km}$ and tens of meters when the range of the target is within $150 \mathrm{~km}$. When the signal-to-noise ratio increases the performance is even better. This performance is to be expected in the case of the radar with large signal bandwidth. However, this performance is good in the case of low-frequency radar, for example when the wavelength of the transmit signal is $15 \mathrm{~m}$ or more, as there is not enough signal bandwidth to provide accurate range estimation. Fortunately, the performance of the 3D coordinate estimation can be achieved without the range information by the proposed scheme. So, the proposed scheme is suitable for lowfrequency radar.

Figure 3 shows the relationship between the 3D coordinate estimation performance and the number of the receive elements, where the element spacing is selected to be a half wavelength. It can be observed that the estimation performance is improved efficiently by increasing the number of receive elements. In the case of the given 

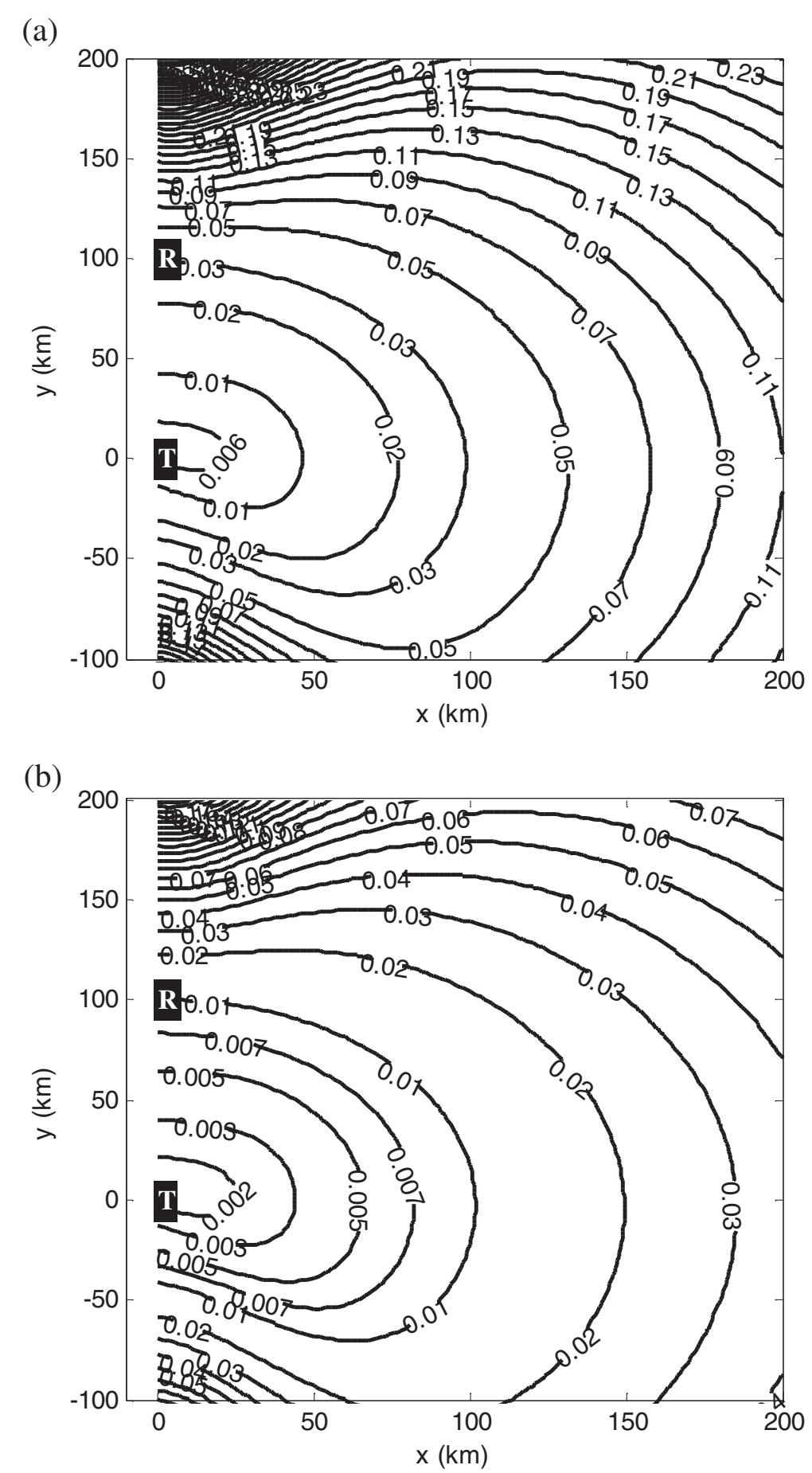

Figure $2 \mathrm{GDOP}$ of the proposed scheme $\left(K=\mathbf{2 0}, \mathbf{N} \mathbf{2 0}, L_{\boldsymbol{b}}=100 \mathbf{k m}=\boldsymbol{r}=\boldsymbol{\lambda}, \boldsymbol{z}=\mathbf{3 5} \mathbf{k m}\right)$. (a) $\mathrm{SNR}=15 \mathrm{~dB}$. (b) $\mathrm{SNR}=25 \mathrm{~dB}$.

element spacing, more elements mean greater aperture. If the spacing is a half-wavelength and the wavelength is $15 \mathrm{~m}$, the aperture would be $150 \mathrm{~m}$ for 20 elements, which implies several technological issues to translate into a real system design. Recently proposed bio-inspired couple compact array can reduce the elements spacing considerably and keep high direction of arrival estimation performance [14]. It is a promising technique to resolve this problem.

\section{Target 3D coordinates estimation method}

In this section, we develop an ESPRIT-like algorithm to estimate the $3 \mathrm{D}$ angles of the targets. 


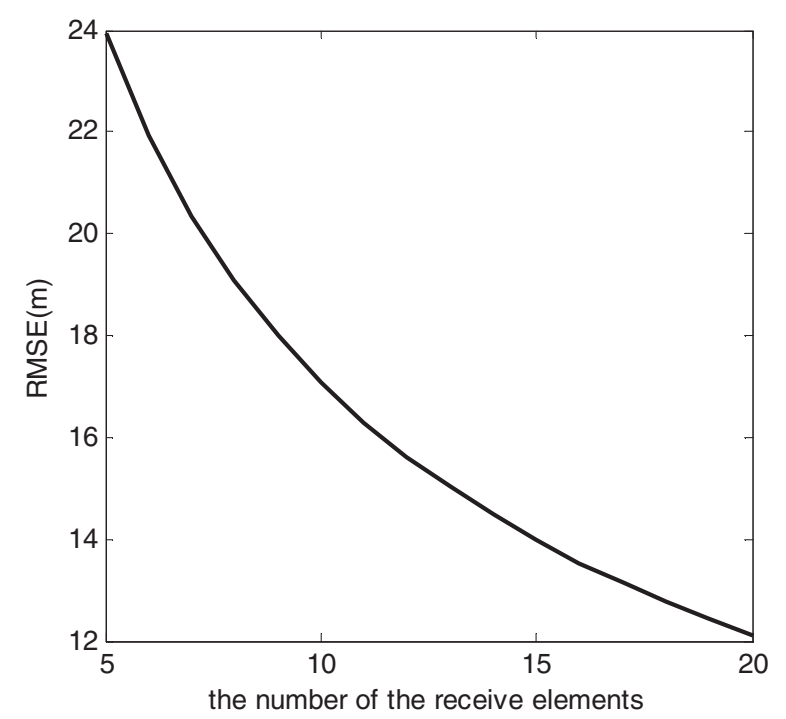

Figure 3 Influence of the number of the receive elements $\left(N=20, L_{b}=100 \mathrm{~km}, r=\lambda\right.$, target: [100 km, $50 \mathrm{~km}$, $35 \mathrm{~km}$, SNR $=25 \mathrm{~dB}$ ).

\subsection{Estimation of the receive cone angles}

To estimate the receive cone angle, we first construct selection matrices $\mathbf{J}_{1}$ and $\mathbf{J}_{2}$.

$$
\begin{aligned}
& \mathbf{J}_{1}=\mathbf{I}_{N} \otimes\left[\mathbf{I}_{K-1}, \mathbf{0}_{(K-1) \times 1}\right] \\
& \mathbf{J}_{2}=\mathbf{I}_{N} \otimes\left[\mathbf{0}_{(K-1) \times 1}, \mathbf{I}_{K-1}\right]
\end{aligned}
$$

where $\mathbf{I}_{K_{-} 1}$ and $\mathbf{I}_{N}$ are identify matrices with size $K-1$ and $N$, respectively. $\mathbf{0}_{\left(K_{-} 1\right) \times 1}$ denotes a zero vector with size $K-1$.

The rotated factor which contain the receive cone angle can be obtained by using selection matrices as follows:

$$
\begin{aligned}
& \mathbf{Y}_{1}=\mathbf{J}_{1} \mathbf{Y}=\mathbf{J}_{1} \mathbf{A H}+\mathbf{J}_{1} \mathbf{W} \\
& \mathbf{Y}_{2}=\mathbf{J}_{2} \mathbf{Y}=\mathbf{J}_{2} \mathbf{A H}+\mathbf{J}_{2} \mathbf{W}=\mathbf{J}_{1} \mathbf{A D H}+\mathbf{J}_{2} \mathbf{W}
\end{aligned}
$$

where the rotated factor is $\mathbf{D}=\operatorname{diag}\left[e^{j \pi \cos \alpha_{1}}, e^{j \pi \cos \alpha_{2}} \ldots\right.$ $\left.e^{j \pi \cos \alpha_{P}}\right]$.

The autocorrelation and crosscorrelation matrices of $\mathbf{Y}_{1}$ and $\mathbf{Y}_{2}$ are as follows:

$$
\begin{aligned}
& \mathbf{R}_{11}=E\left[\mathbf{Y}_{1} \mathbf{Y}_{1}{ }^{H}\right]=\mathbf{J}_{1} \mathbf{A} \mathbf{R}_{s} \mathbf{A}^{H} \mathbf{J}_{1}{ }^{H}+\mathbf{R}_{W 1} \\
& \mathbf{R}_{21}=E\left[\mathbf{Y}_{2} \mathbf{Y}_{1}{ }^{H}\right]=\mathbf{J}_{1} \mathbf{A D} \mathbf{R}_{s} \mathbf{A}^{H} \mathbf{J}_{1}{ }^{H}
\end{aligned}
$$

where $\mathbf{R}_{s}=E\left[\mathbf{H H}^{H}\right]$ and $\mathbf{R}_{W 1}=\sigma^{2} \mathbf{I}_{N K-1}$. As proved in [11], the noise covariance $\sigma$ here is the same as that at the MIMO radar receiver. It can be obtained when there is no input signal in the receiver. So the effect of noise can be cancelled as follows:

$$
\mathbf{R}_{11 s}=\mathbf{R}_{11}-\sigma^{2} \mathbf{I}_{N K-1}=\mathbf{J}_{1} \mathbf{A} \mathbf{R}_{s} \mathbf{A}^{H} \mathbf{J}_{1}{ }^{H}
$$

Define $\mathbf{A}_{r}=\mathbf{J}_{1} \mathbf{A}$ and construct the matrix $\mathbf{R}=\mathbf{R}_{21} \mathbf{R}_{11 s}^{\#}$, where $\mathbf{R}_{11 s}^{\#}$ is the Penrose-Moore inverse of $\mathbf{R}_{11 s}$. Just as in the method proposed in [15], we can write

$$
\mathbf{R} \mathbf{A}_{r}=\mathbf{A}_{r} \mathbf{D}
$$

Estimates of the receive cone angles are achieved via eigendecomposition of $\mathrm{R}$ as

$$
\mathbf{R}=\mathbf{U} \boldsymbol{\Lambda} \mathbf{U}^{H}
$$

where $\Lambda=\operatorname{diag}\left[\lambda_{1}, \lambda_{2}, \cdots, \lambda_{P}\right]$ is constructed by the maximum $P$ eigenvalue of $\mathbf{R}$. The number of targets $P$ should be estimated in advance. The issue of the target number detection for bistatic MIMO radar can be found in [16]. From (21) and (22), the receive angle of the $p$ th target is

$$
\alpha_{p}=\arccos \left(\frac{\operatorname{angle}\left(\lambda_{i}\right)}{\pi}\right)
$$

\subsection{Estimation of the transmit angles}

From (21) and (22), we can obtain that $\operatorname{span}\left\{\mathbf{A}_{r}\right\}=$ span $\{\mathbf{U}\}$. So the transmit angle information can be extracted from $\mathbf{U}=\left[\mathbf{u}_{1}, \cdots, \mathbf{u}_{P}\right]$. The vector $\tilde{\mathbf{a}}$ should be first constructed to separate the transmit angle information of the $p$ th target from the matrix $\mathbf{u}_{p}$ as follows:

$$
\begin{aligned}
& \tilde{\mathbf{a}}\left(\alpha_{p}\right)=\operatorname{kron}([1, \ldots, 1])^{T}, \mathbf{a}_{r}\left(\alpha_{p}\right) \\
& \tilde{\mathbf{y}}_{p}=\mathbf{u}_{p} \odot \tilde{\mathbf{a}}\left(\alpha_{p}\right)
\end{aligned}
$$

where $\odot$ denotes hadamard product and $\mathbf{a}_{r}\left(\alpha_{p}\right)$ is a vector constructed by the first $K-1$ elements of $\mathbf{a}\left(\alpha_{p}\right)$. Divide $\tilde{\mathbf{y}}_{p}$ into $K-1$ vectors with the size of $N \times 1$ and average the vectors as follow:

$$
\bar{y}_{p}=\frac{1}{K-1} \sum_{k=1}^{K-1}\left[\tilde{y}_{k}, \tilde{y}_{(K-1)+k} \cdots, \tilde{y}_{(N-1)(K-1)+k}\right]^{T}
$$

Then UCA-ESPRIT algorithm can be used to estimate the transmit azimuth angle and elevation angle. The phase mode excitation method is exploited to simplify the array manifold of the circular array. The beamformer matrix $\mathbf{F}_{r}^{H}$ is constructed to transform the UCA manifold vector to the beamspace manifold [17].

$$
\mathbf{F}_{r}^{H}=\mathbf{G}^{H} \mathbf{C}_{v} \mathbf{V}^{H}
$$

where $\mathbf{C}_{v}=\operatorname{diag}\left(j^{-M}, \ldots j^{-1}, j^{0}, j^{-1}, \ldots . j^{-M}\right) . \quad M \approx \frac{2 \pi r}{\lambda}$ is the highest order mode that can be excited by the aperture 
at a reasonable strength. $\mathbf{G}=\frac{1}{\sqrt{2 M+1}}\left[\mathbf{w}\left(a_{-M}\right), \ldots, \mathbf{w}\left(a_{0}\right)\right.$, $\left.\ldots, \mathbf{w}\left(a_{M}\right)\right]$, where $a_{m}=\frac{2 \pi m}{2 M+1}, m \in[-M, M]$ and $\left(a_{m}\right)=$ $\left[e^{-j M a_{m}}, \ldots, e^{-j a_{m}}, e^{j 0 a_{m}}, e^{j a_{m}}, \ldots, e^{j M a_{m}}\right]^{T} . \mathbf{V}=\frac{1}{\sqrt{N}}\left[v_{-M}, \ldots v_{0}\right.$, $\left.\ldots v_{M}\right]$, where $\mathbf{v}_{m}=\left[1, e^{j 2 \pi m / N}, \ldots, e^{j 2 \pi m(N-1) / N}\right]^{H}$.

The selected data after transformation is as follows:

$$
\mathbf{y}_{p}=\mathbf{F}_{r}^{H} \bar{y}_{p}
$$

The sample covariance matrix can be calculated as follows:

$$
\mathbf{R}_{p}=\frac{1}{L} \mathbf{y}_{p} \mathbf{y}_{p}^{H}, \quad p=1,2, \ldots, P
$$

EVD of the matrix $\operatorname{Re}\left(\mathbf{R}_{p}\right)$ is performed to obtain the real-value signal subspace $\mathbf{s}_{p}$ which is the eigenvectors of the largest eigenvalue of the matrix $\operatorname{Re}\left(\mathbf{R}_{p}\right) \cdot \operatorname{Re}(\bullet)$ denotes the real part of the elements of the matrix. $P$ times EVD should be performed separately to estimate $P$ eigenvector.

Transform the real-value signal subspace as follows:

$$
\mathbf{s}_{0}=\mathbf{C}_{0} \mathbf{W} \mathbf{s}_{p}
$$

where $\mathbf{C}_{0}=\operatorname{diag}\{(-1)^{N}, \ldots,(-1)^{1}, \overbrace{1,1, \ldots, 1}^{M+1}\}$. Define $\mathbf{s}_{0}^{(-1)}$ and $\mathbf{s}_{0}^{(0)}$ as the first and the last $2 M-1$ elements of the vector $\mathbf{s}_{0}$ respectively. The following equation can be founded [17]

$$
E \hat{u}=\Gamma \mathbf{s}_{0}^{(0)}
$$

where $\mathbf{E}=\left[\begin{array}{ll}\mathbf{s}_{0}{ }^{(-1)} & \mathbf{D} \hat{\mathbf{I}} \mathbf{s}_{0}^{(-1)_{*}}\end{array}\right]$ and $\boldsymbol{\Gamma}=\frac{\lambda}{\pi r} \operatorname{diag}\{-(M-1)$, $\ldots, 0, \ldots,(M-1)\} . D=\operatorname{diag}\left\{(-1)^{M-2}, \ldots,(-1)^{1},(-1)^{0},(-1)^{1}\right.$, $\left.\ldots,(-1)^{M}\right\}$ and $\hat{\mathbf{I}}$ is the reverse permutation matrix with ones on the anti-diagonal and zeros elsewhere. The least square solution of (30) is $\hat{\mathbf{u}}_{L S}=\left[\begin{array}{ll}\hat{u}_{p} & \hat{u}_{p}^{*}\end{array}\right]^{T}$.

The transmit azimuth angles and elevation angles can be obtained from $\hat{u}_{p}, p=1,2, \ldots P$, as $\hat{u}_{p}=\sin \theta_{p} e^{j \varphi_{p}}$ [17].

So, the azimuth angles $\phi_{p}$ and elevation angles $\theta_{p}$ can be calculated from the following formulas.

$$
\begin{aligned}
& \varphi_{p}=\arg \left(\hat{u}_{p}\right) \\
& \theta_{p}=\arcsin \left|\hat{u}_{p}\right|
\end{aligned}
$$

The receive angles and transmit angles can be paired automatically as they are connected by the corresponding eigenvalues and eigenvectors, respectively.

\subsection{D coordinates of the targets}

In this section, the $3 \mathrm{D}$ coordinates are calculated by the angles estimated in Sections 4.1 and 4.2. The relationship between $[\alpha, \varphi, \theta]$ and $3 \mathrm{D}$ coordinates of the target $[x, y, z]$ can be found by the geometry of bistatic MIMO radar in Figure 1 as follows:

$$
\begin{aligned}
& x=\frac{L_{b} \sin \theta \cos \varphi}{\mathrm{c} \tan \alpha \sqrt{1-\sin ^{2} \theta \sin ^{2} \varphi}+\sin \theta \sin \varphi} \\
& y=\frac{L_{b} \sin \theta \sin \varphi}{\mathrm{ctan} \alpha \sqrt{1-\sin ^{2} \theta \sin ^{2} \varphi}+\sin \theta \sin \varphi} \\
& z=\frac{L \cos \theta}{\mathrm{ctan} \alpha \sqrt{1-\sin ^{2} \theta \sin ^{2} \varphi}+\sin \theta \sin \varphi}
\end{aligned}
$$

It can be observed that the $3 \mathrm{D}$ coordinates of the targets are determined by the 3D angles estimated above and the baseline of the bistatic radar.

\section{Simulation and analysis}

In this section, we demonstrate via simulations the performances of the proposed scheme. As shown in Figure 1, a transmit UCA with radius $r=\lambda$ is employed for the simulations and the number of the transmit elements is selected as $N=20$. The receive array is a ULA with 20 elements spaced at a half-wavelength. The baseline between transmitter and receiver is $100 \mathrm{~km}$. In these simulations, we assume a low-frequency radar system. The transmit signals are narrowband and centered at $20 \mathrm{MHz}(\lambda=15 \mathrm{~m})$. We first estimate the transmit azimuth angle, transmit elevation angle, and receive cone angle by the proposed method and then calculate the $3 \mathrm{D}$ coordinates of the targets according to the angles. The localization performance is evaluated by the RMSE of the estimated values. Five hundreds Monte Carlo trials are performed.

\subsection{Simulation 1: the influence of the SNR}

The influence of SNR for the coordinate estimation is studied in the case of two targets. The targets are located at $(73.5 \mathrm{~km}, 34.094 \mathrm{~km}, 35 \mathrm{~km})$ and $(65.5 \mathrm{~km}$, $7.8932 \mathrm{~km}, 35 \mathrm{~km}$ ) respectively. The range cell of the targets is at $193 \mathrm{~km}$. The normalized Doppler frequencies of two targets are selected as 0.1 and 0.9 respectively. The performance of both the angles and the coordinate estimation are shown in Figure 4. The performances of the estimated angles of two targets are plotted in Figure $4 \mathrm{a}, \mathrm{b}$, respectively. It is shown that the performance of the proposed ESPRIT algorithm can be improved by the increase of the SNR. Figure 4c shows the $3 \mathrm{D}$ coordinates estimate performance of the two targets. It is shown that the locate accuracy approach wavelength of the transmit signal by using proposed ESPRIT-based algorithm and the proposed scheme has the potential to 
(a)

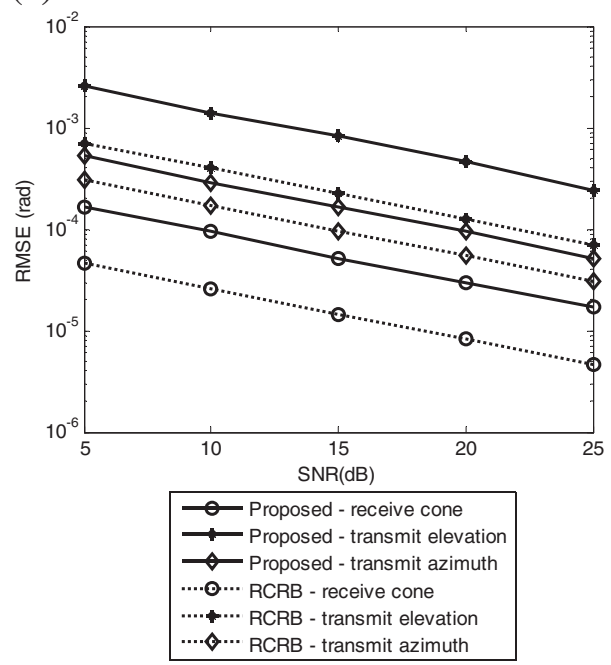

(b)

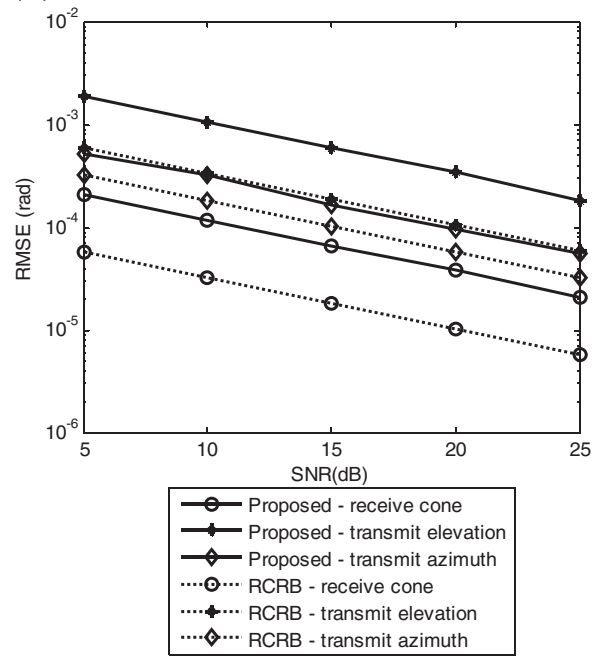

(c)

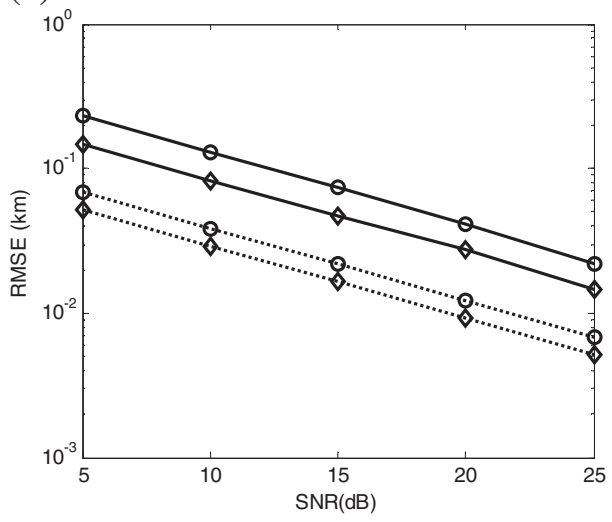

- Proposed - target 1

........ Bound - target 1

$\checkmark$ - Proposed - target 2

$\cdots$.... Bound - target 2
Figure 4 Localization performance v.s SNR $(K=20, N=20$,

$L_{b}=100 \mathrm{~km}, r=\lambda$ ). (a) performance of the angle estimation of the targets 1. (b) performance of the angle estimation of the target 2.

(c) 3D coordinates estimate error.

achieve meter-level locate accuracy which is far less than the wavelength of the transmit signal. It can be observed that the estimation performances of the two targets are different from each other as the localization performance of the bistatic radar is related to location of the targets.

\subsection{Simulation 2: the identifiability of the adjacent targets}

The identifiability of two adjacent targets is investigated by the simulation. Targets 1 and 2 are at the same range cell in the plane $z=35 \mathrm{~km}$. Target 1 is located at $(73.5 \mathrm{~km}, 34.094 \mathrm{~km}, 35 \mathrm{~km})$. Target 2 changes its location along the isorange ellipse of bistatic radar as shown in Figure 5. The $\Delta x$ in Figure 6 denotes the difference of the $x$-coordinate between targets 1 and 2 . The normalized Doppler frequencies of the two targets are selected as 0.1 and 0.9, respectively. It is shown in Figure 6(a)(b) that the estimation performance of target 1 is improved when target 2 is far away from it by using the proposed algorithm. However, the influence of the target 2 can be ignored when $\Delta x>0.4 \mathrm{~km}$. It can also be observed that the estimation performance of target 1 declines considerably when $\Delta x$ is within $0.05 \mathrm{~km}$. The results imply the identifiability of the two adjacent targets by using the proposed algorithm. While the RMSE of the proposed algorithm tends to increase in proximity of the $x$-axis origin, the RCRB maintains flat behavior. The reason is that the proposed algorithm can only distinguish the

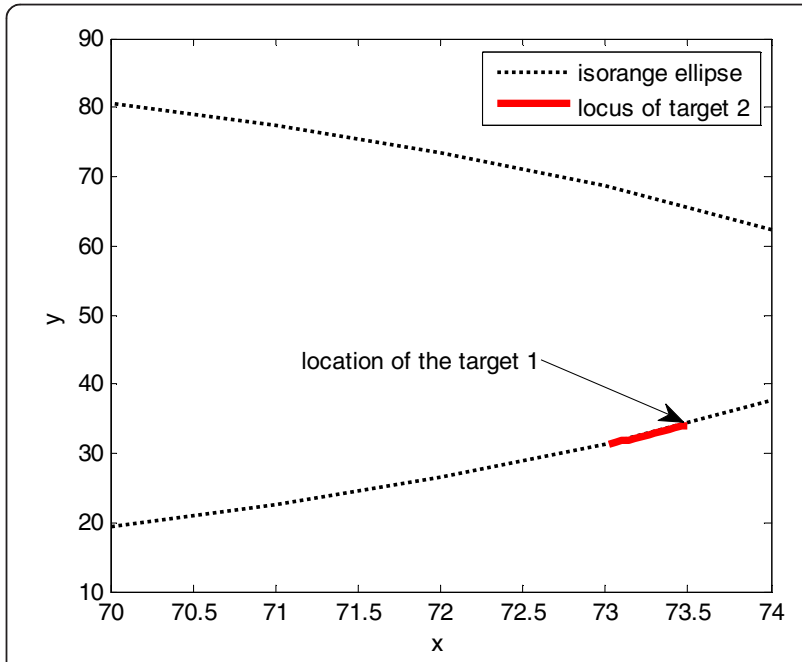

Figure 5 The location relationship between target 1 and target 2. 


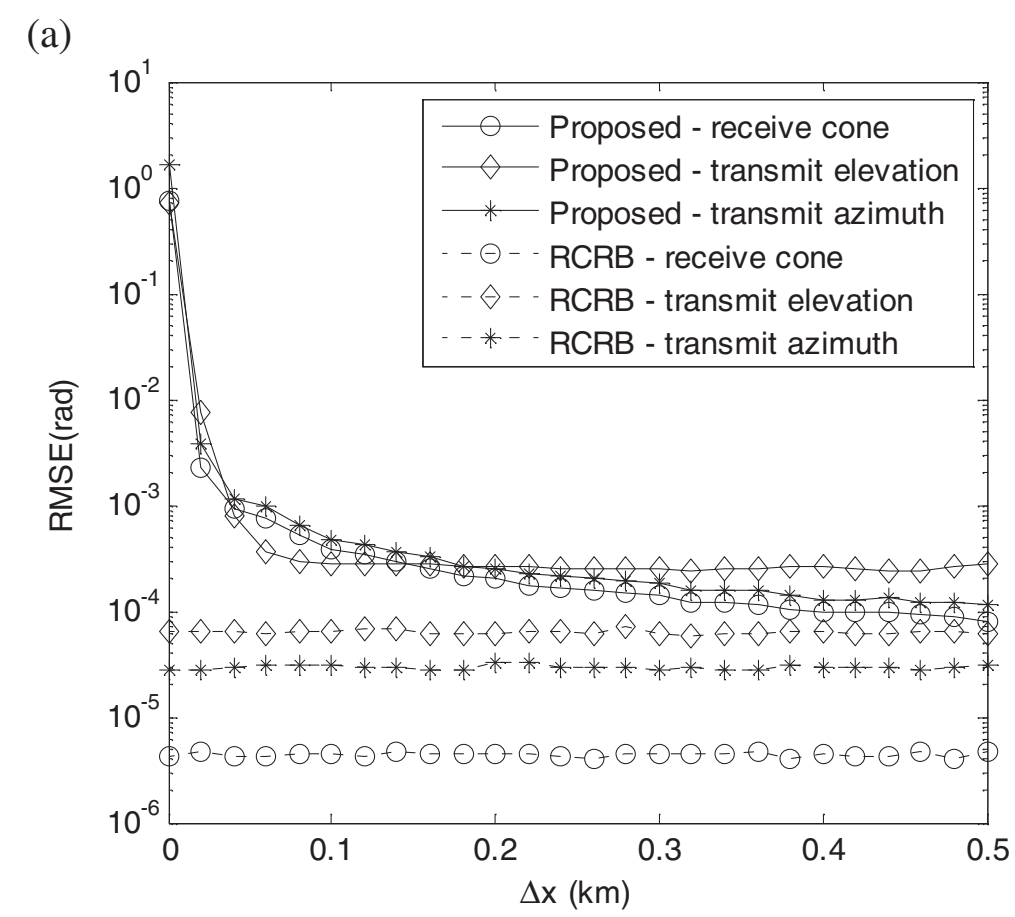

(b)

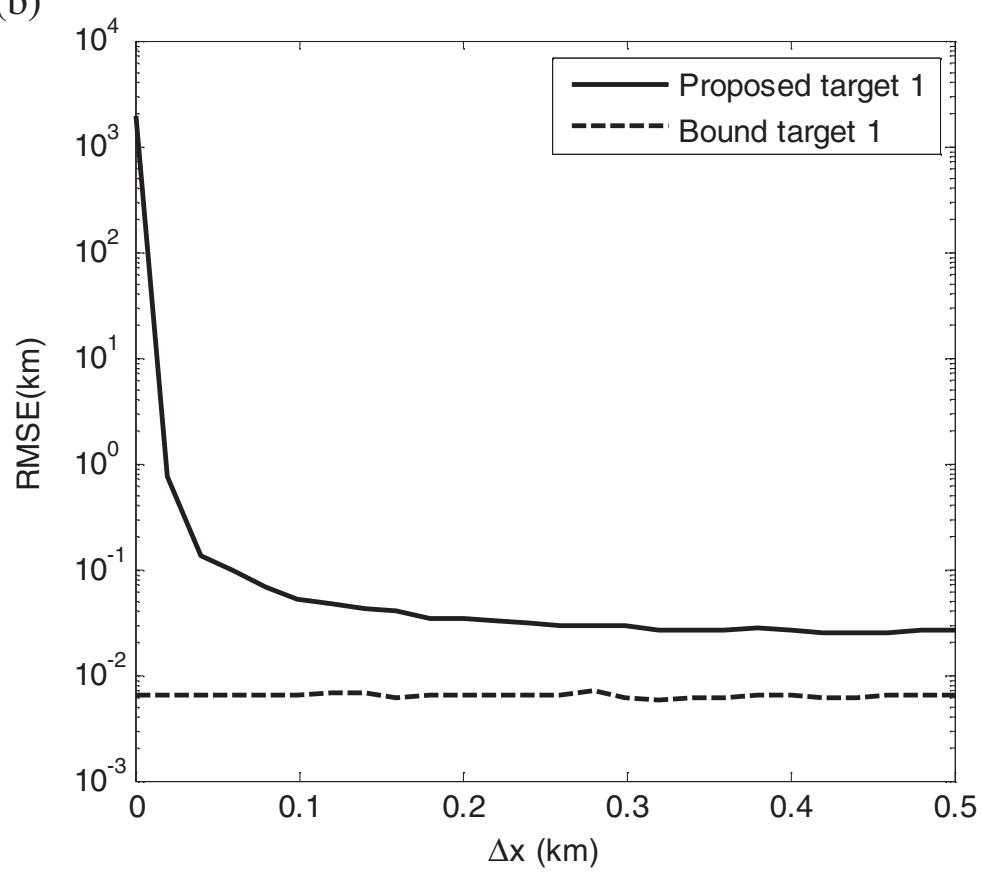

Figure 6 Identifiability of adjacent targets $\left(K=20, N=20, L_{b}=100 \mathrm{~km}, r=\lambda\right.$, SNR $=25 \mathrm{~dB}$, target 1 at $(73.5 \mathrm{~km}, 34.094 \mathrm{~km}, 35 \mathrm{~km})$, $\boldsymbol{f}_{\boldsymbol{D}}=[\mathbf{0 . 1}, \mathbf{0 . 9}]$ ). (a) angle estimation performance of target 1. (b) 3D coordinates estimation performance of target 1 .

two targets by their angles. However, there still are other different characteristic of the two targets, such as reflection coefficient and Doppler frequency. The behavior of the RCRB implies that the targets can be distinguished by these characteristic in theory. Furthermore, Equation (38) in Appendix 1 discloses the behaviour of the RCRB in mathematical terms. It can be observed that the Fisher submatrix is a Hadamard product of $\dot{\mathbf{A}}_{\boldsymbol{\alpha}}^{H} \mathbf{R}_{\mathrm{W}}^{-1} \dot{\mathbf{A}}_{\boldsymbol{\alpha}}$ and $\mathbf{R}_{s}^{T}$, where $\dot{\mathbf{A}}_{\boldsymbol{\alpha}}^{H} \mathbf{R}_{\mathrm{W}}^{-1} \dot{\mathbf{A}}_{\boldsymbol{\alpha}}$ contains the angle information and $\mathbf{R}_{s}^{T}$ contains the reflection coefficient and Doppler frequency of the targets. Full rank of $\mathbf{R}_{s}^{T}$ can guarantee 
the full rank of the Fisher Matrix, even though the matrix $\dot{\mathbf{A}}_{\alpha}^{H} \mathbf{R}_{\mathrm{W}}^{-1} \dot{\mathbf{A}}_{\boldsymbol{\alpha}}$ tends to rank defect in the case of two adjacent targets.

\subsection{Simulation 3: influence of target range}

The relationship between the estimation performances and target range is investigated in this subsection. Assume the $x$ and $z$ coordinates of the target are fixed at $x=50 \mathrm{~km}$ and $z=35 \mathrm{~km}$ respectively. The target location is changed along the $y$-axis from $50 \mathrm{~km}$ to $300 \mathrm{~km}$. The signal-to-noise ratio is $25 \mathrm{~dB}$. Figure 7 (a) and (b) plots the performances of the angle and corresponding $3 \mathrm{D}$ coordinate estimation respectively. The dashed lines are the results of the proposed algorithms and the solid lines are the RCRB. It is shown that from both the proposed algorithm and the RCRB that the angle estimation performances vary little with the target range. However, the performance of the 3D coordinate estimation degrades with increasing target range. This simulation result is consistent with the analysis of the GDOP in Section 3.3. It seems that the proposed scheme is suitable to locate the target at relatively short range.

\section{Conclusions}

The transmit UCA and receive ULA configuration scheme for bistatic MIMO radar have been proposed to achieve target 3D localization. The performance bound of this scheme is evaluated and an ESPRIT-like algorithm was developed to achieve the 3D coordinate estimation of multiple targets. The advantage of the proposed scheme is that the 3D coordinates of multiple targets can be estimated without the range information and has the capability for identification of multiple targets in the same range cell. Moreover, it is suitable for lowfrequency radars to estimate the location of relatively short range targets. How to reduce the receive element spacing as well as keeping high angle estimation performance is the focus of our future work.

\section{Appendix}

\section{A 1: Derivation of the FIM}

In this section, the submatrices of FIM in (9) are derived.

The $(i, j)$ th elements of the submatrix $\mathbf{F}_{1,1}$ are [12]

$$
\begin{aligned}
\mathbf{F}_{1,1}\left(\alpha_{i}, \alpha_{j}\right) & =2 \operatorname{Re}\left\{\operatorname{tr}\left\{\left(\frac{\partial \mathbf{A}(\boldsymbol{\alpha}, \varphi, \boldsymbol{\theta}) \mathbf{H}}{\partial \alpha_{i}}\right)^{H} \mathbf{R}_{\mathbf{W}}^{-1}\left(\frac{\partial \mathbf{A}(\boldsymbol{\alpha}, \varphi, \boldsymbol{\theta}) \mathbf{H}}{\partial \alpha_{j}}\right)\right\}\right\} \\
= & 2 \operatorname{Re}\left\{\operatorname{tr}\left\{\left(\dot{\mathbf{A}}_{\boldsymbol{\alpha}} \mathbf{e}_{i} \mathbf{e}_{i}^{T} \mathbf{H}\right)^{H} \mathbf{R}_{\mathbf{W}}^{-1}\left(\dot{\mathbf{A}}_{\boldsymbol{\alpha}} \mathbf{e}_{\mathbf{e}} \mathbf{e}_{j}^{T} \mathbf{H}\right)\right\}\right\} \\
& =2 \operatorname{Re}\left\{\left(\mathbf{e}_{i}^{T} \dot{\mathbf{A}}_{\boldsymbol{\alpha}}^{H} \mathbf{R}_{\mathrm{W}}^{-1} \dot{\mathbf{A}}_{\boldsymbol{\alpha}} \mathbf{e}_{j}\right)\left(\mathbf{e}_{j}^{T} \mathbf{H H}^{H} \mathbf{e}_{i}\right)\right\} \\
& =\frac{2 L}{\sigma^{2}} \operatorname{Re}\left\{\left(\dot{\mathbf{A}}_{\boldsymbol{\alpha}}^{H} \dot{\mathbf{A}}_{\boldsymbol{\alpha}}\right)_{i, j}\left(\mathbf{R}_{s}^{T}\right)_{i, j}\right\}
\end{aligned}
$$

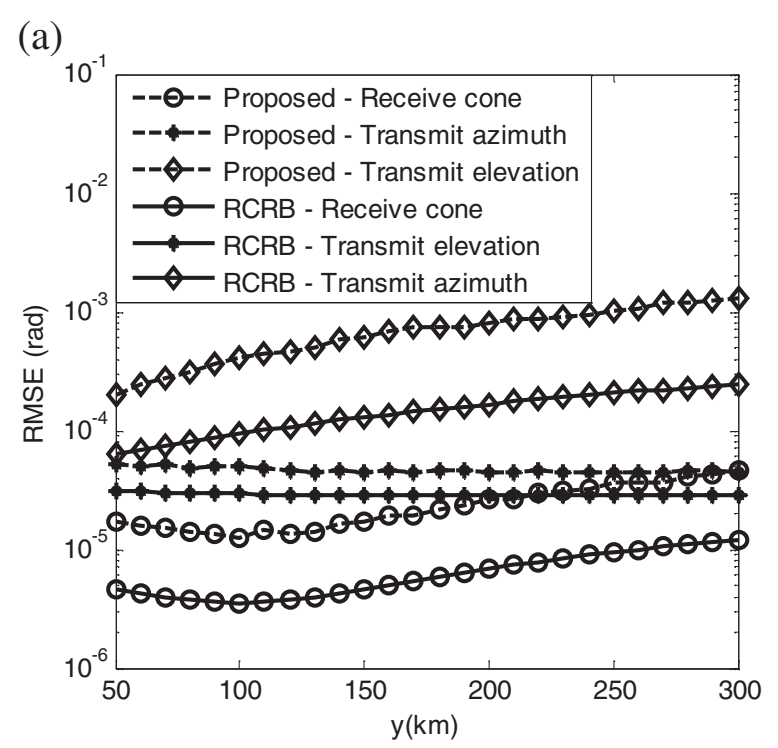

(b)

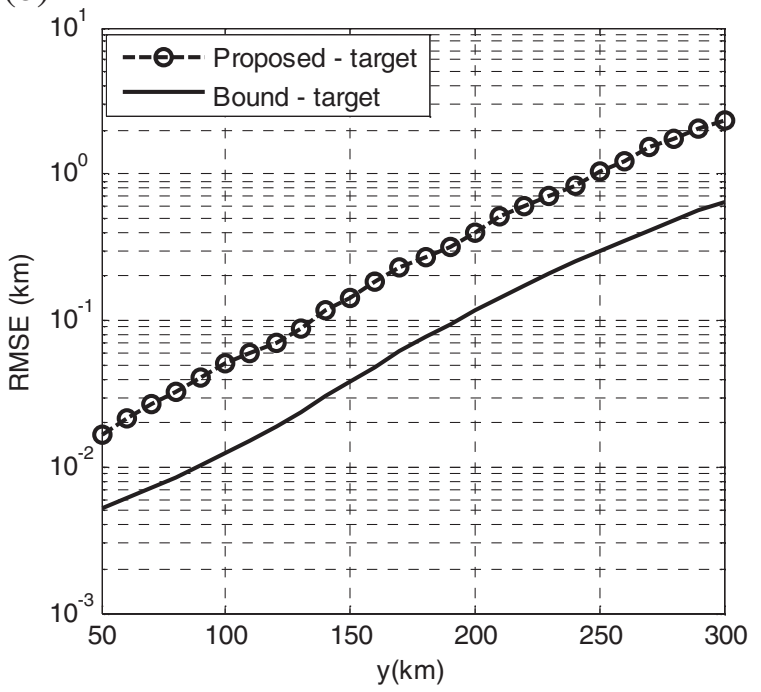

Figure 7 Influence of target range $\left(K=20, N=20, L_{b}=100 \mathrm{~km}\right.$, $r=\lambda, \mathrm{SNR}=\mathbf{2 5 \mathrm { dB }}, \boldsymbol{x}=\mathbf{5 0} \mathrm{km}$ and $\boldsymbol{z}=\mathbf{3 5} \mathrm{km}$ ). (a) angle estimation performance of the target. (b) $3 \mathrm{D}$ coordinates estimation performance of the target.

where $\dot{\mathbf{A}}_{\boldsymbol{\alpha}}=\left[\mathbf{b}\left(\varphi_{1}, \theta_{1}\right) \otimes \frac{\partial \mathbf{a}\left(\alpha_{1}\right)}{\partial \alpha_{1}} \mathbf{b}\left(\varphi_{2}, \theta_{2}\right) \otimes \frac{\partial \mathbf{a}\left(\alpha_{2}\right)}{\partial \alpha_{2}} \cdots \mathbf{b}\right.$ $\left.\left(\varphi_{P}, \theta_{P}\right) \otimes \frac{\partial \mathbf{a}\left(\alpha_{P}\right)}{\partial \alpha_{P}}\right] . \mathbf{R}_{n}=\sigma^{2} \mathbf{I}_{K}$ and $\mathbf{R}_{\mathrm{W}}=\mathbf{I}_{N} \otimes \mathbf{R}_{n} . \otimes$ denotes the kronecker product. $\operatorname{Re}\{g\}$ denotes the real part of the data and $\operatorname{tr}\{\mathrm{g}\}$ denotes the trace of the matrix. $\mathbf{e}_{i}$ denotes a column vector with 1 at the ith element and zeros at the others. So we can obtain

$$
\mathbf{F}_{1,1}=2 L \operatorname{Re}\left\{\left(\dot{\mathbf{A}}_{\boldsymbol{\alpha}}^{H} \mathbf{R}_{\mathrm{W}}^{-1} \dot{\mathbf{A}}_{\boldsymbol{\alpha}}\right) \odot\left(\mathbf{R}_{s}^{T}\right)\right\}
$$


In a similar way to the derivation above, we can obtain the other submatrices of the FIM as follows:

$$
\begin{aligned}
& \mathbf{F}_{1,2}=\frac{2 L}{\sigma^{2}} \operatorname{Re}\left\{\left(\dot{\mathbf{A}}_{\boldsymbol{\alpha}}^{H} \dot{\mathbf{A}}_{\boldsymbol{\varphi}}\right) \odot\left(\mathbf{R}_{s}^{T}\right)\right\} \\
& \mathbf{F}_{1,3}=\frac{2 L}{\sigma^{2}} \operatorname{Re}\left\{\left(\dot{\mathbf{A}}_{\boldsymbol{\alpha}}^{H} \dot{\mathbf{A}}_{\boldsymbol{\theta}}\right) \odot\left(\mathbf{R}_{s}^{T}\right)\right\} \\
& \mathbf{F}_{2,1}=\frac{2 L}{\sigma^{2}} \operatorname{Re}\left\{\left(\dot{\mathbf{A}}_{\boldsymbol{\varphi}}^{H} \dot{\mathbf{A}}_{\boldsymbol{\alpha}}\right) \odot\left(\mathbf{R}_{s}^{T}\right)\right\} \\
& \mathbf{F}_{2,2}=\frac{2 L}{\sigma^{2}} \operatorname{Re}\left\{\left(\dot{\mathbf{A}}_{\boldsymbol{\varphi}}^{H} \dot{\mathbf{A}}_{\boldsymbol{\varphi}}\right) \odot\left(\mathbf{R}_{s}^{T}\right)\right\} \\
& \mathbf{F}_{2,3}=\frac{2 L}{\sigma^{2}} \operatorname{Re}\left\{\left(\dot{\mathbf{A}}_{\boldsymbol{\varphi}}^{H} \dot{\mathbf{A}}_{\boldsymbol{\theta}}\right) \odot\left(\mathbf{R}_{s}^{T}\right)\right\} \\
& \mathbf{F}_{3,1}=\frac{2 L}{\sigma^{2}} \operatorname{Re}\left\{\left(\dot{\mathbf{A}}_{\boldsymbol{\theta}}^{H} \dot{\mathbf{A}}_{\boldsymbol{\alpha}}\right) \odot\left(\mathbf{R}_{s}^{T}\right)\right\} \\
& \mathbf{F}_{3,2}=\frac{2 L}{\sigma^{2}} \operatorname{Re}\left\{\left(\dot{\mathbf{A}}_{\boldsymbol{\theta}}^{H} \dot{\mathbf{A}}_{\boldsymbol{\varphi}}\right) \odot\left(\mathbf{R}_{s}^{T}\right)\right\} \\
& \mathbf{F}_{3,3}=\frac{2 L}{\sigma^{2}} \operatorname{Re}\left\{\left(\dot{\mathbf{A}}_{\boldsymbol{\theta}}^{H} \dot{\mathbf{A}}_{\boldsymbol{\theta}}\right) \odot\left(\mathbf{R}_{s}^{T}\right)\right\}
\end{aligned}
$$

where $\quad \dot{\mathbf{A}}_{\boldsymbol{\theta}}=\left[\frac{\partial \mathbf{b}\left(\varphi_{1}, \theta_{1}\right)}{\partial \theta_{1}} \otimes \mathbf{a}\left(\alpha_{1}\right) \frac{\partial \mathbf{b}\left(\varphi_{2}, \theta_{2}\right)}{\partial \theta_{2}} \otimes \mathbf{a}\left(\alpha_{2}\right) \cdots\right.$ $\left.\frac{\partial \mathbf{b}\left(\varphi_{P}, \theta_{P}\right)}{\partial \theta_{P}} \otimes \mathbf{a}\left(\alpha_{P}\right)\right] \quad$ and $\quad \dot{\mathbf{A}}_{\varphi}=\left[\frac{\partial \mathbf{b}\left(\varphi_{1}, \theta_{1}\right)}{\partial \varphi_{1}} \quad \otimes \mathbf{a}\left(\alpha_{1}\right)\right.$ $\left.\frac{\partial \mathbf{b}\left(\varphi_{2}, \theta_{2}\right)}{\partial \varphi_{2}} \otimes \mathbf{a}\left(\alpha_{2}\right) \cdots \frac{\partial \mathbf{b}\left(\varphi_{P}, \theta_{P}\right)}{\partial \varphi_{P}} \otimes \mathbf{a}\left(\alpha_{P}\right)\right]$.

\section{Appendix 2. derivation of the error propagation} matrix

The relationships between the 3D coordinates and the $3 \mathrm{D}$ angles are as follows:

$$
\begin{aligned}
& \alpha=\arccos \left(\frac{L_{b}-y}{\sqrt{x^{2}+\left(L_{b}-y\right)^{2}+z^{2}}}\right) \\
& \varphi=\arctan \left(\frac{y}{x}\right) \\
& \theta=\arctan \left[\frac{\sqrt{x^{2}+y^{2}}}{z}\right]
\end{aligned}
$$

The estimate error in $\left[\alpha_{0}, \theta_{0}, \phi_{0}\right]^{T}$ can be obtained by expanding the angles $[\alpha, \theta, \phi]^{T}$ about the point $\left[x_{0}, y_{0}, z_{0}\right]^{T}$ in a Taylor series. The first term in Taylor expansion gives [13].

$$
\Delta \mathbf{v}=\mathbf{T} \Delta \mathbf{e}
$$

where

$$
\mathbf{T}=\left[\begin{array}{lll}
\frac{\partial \alpha}{\partial x} & \frac{\partial \alpha}{\partial y} & \frac{\partial \alpha}{\partial z} \\
\frac{\partial \varphi}{\partial x} & \frac{\partial \varphi}{\partial y} & \frac{\partial \varphi}{\partial z} \\
\frac{\partial \theta}{\partial x} & \frac{\partial \theta}{\partial y} & \frac{\partial \theta}{\partial z}
\end{array}\right]
$$

The elements of the error propagation matrix $\mathrm{C}$ are as follows:

$$
\begin{aligned}
& \frac{\partial \alpha}{\partial x}=\frac{x\left(L_{b}-y\right)}{\left[x^{2}+\left(L_{b}-y\right)^{2}+z^{2}\right] \sqrt{x^{2}+z^{2}}} \\
& \frac{\partial \alpha}{\partial y}=\frac{\sqrt{x^{2}+z^{2}}}{x^{2}+\left(L_{b}-y\right)^{2}+z^{2}} \\
& \frac{\partial \alpha}{\partial z}=\frac{\left(L_{b}-y\right) z}{\left[x^{2}+\left(L_{b}-y\right)^{2}+z^{2}\right] \sqrt{x^{2}+z^{2}}} \\
& \frac{\partial \varphi}{\partial x}=\frac{-y}{x^{2}+y^{2}} \\
& \frac{\partial \varphi}{\partial y}=\frac{x}{x^{2}+y^{2}} \\
& \frac{\partial \varphi}{\partial z}=0 \\
& \frac{\partial \theta}{\partial x}=\frac{x z}{\left[x^{2}+y^{2}+z^{2}\right] \sqrt{x^{2}+y^{2}}} \\
& \frac{\partial \theta}{\partial y}=\frac{y z}{\left[x^{2}+y^{2}+z^{2}\right] \sqrt{x^{2}+y^{2}}} \\
& \frac{\partial \theta}{\partial z}=\frac{-\sqrt{x^{2}+y^{2}}}{x^{2}+y^{2}+z^{2}}
\end{aligned}
$$

\section{Competing interests}

The authors declare that they have no competing interests.

\section{Acknowledgments}

This study has been supported by the National Natural Science Foundation of China under contract No. 61271292 and the Fundamental Research Funds for the Central Universities. The authors are grateful to the anonymous referees for their constructive comments and suggestions in improving the quality of this paper.

\section{Author details}

${ }^{1}$ National Lab of Radar Signal Processing, Xidian University, Xi'an 710071,

China. ${ }^{2}$ Department of Electronic and Electrical Engineering, University

College London, London WC1E 6BT, UK.

Received: 31 July 2012 Accepted: 26 February 2013 Published: 17 April 2013 


\section{References}

1. A Haimovich, R Blum, L Cimini, MIMO radar with widely separated antennas. IEEE Signal Processing Magazine 25, 116-129 (2008)

2. L Jian, P Stoica, MIMO radar with colocated antennas. IEEE Signal Processing Magazine 24, 106-114 (2007)

3. Y Haidong, L Jun, L Guisheng, "Multitarget identification and localization using bistatic MIMO radar systems. EURASIP Journal on Advances in Signal Processing 2008, 8 (2008). Article ID 283483

4. NJ Willis, HD Griffiths, Advances in Bistatic Radar (SciTech Publishing, Raleigh, NC, 2007)

5. M Jin, G Liao, J Li, Joint DOD and DOA estimation for bistatic MIMO radar. Signal Processing 89(2), 244-251 (2009)

6. J Chen, G Hong, S Weimin, A new method for joint DOD and DOA estimation in bistatic MIMO radar. Signal Processing 90(2), 714-718 (2010)

7. D Nion, ND Sidiropoulos, Adaptive algorithms to track the PARAFAC decomposition of a third-order tensor. IEEE Trans. on Signal Processing 57(6), 2299-2310 (2009)

8. ML Bencheikh, W Yide, H Hongyang, "Polynomial root finding technique for joint DOA DOD estimation in bistatic MIMO radar. Signal Processing 90(no.9), 2723-2730 (2010)

9. XF Zhang, ZY Xu, LY Xu, DZ Xu, "Trilinear Decomposition-based Transmit Angle and Receive Angle Estimation for MIMO radar", IET Radar. Sonar \& Navig. 5(6), 626-631 (2011)

10. X Zhang, X Gao, G Feng, D Xu, Blind joint DOA and DOD estimation and identifiability results for MIMO radar with different transmit/receive array manifolds. Progress in Electromagnetics Research B 18, 101-109 (2009)

11. L Jian, X Luzhou, P Stoica, KW Forsythe, DW Bliss, Range compression and waveform optimization for MIMO radar: a Cramer-Rao bound based study. IEEE Trans. on Signal Processing 50(no.1), 218-232 (2008)

12. P Stoica, RL Moses, Spectral Analysis of Signals (Prentice-Hall, N.J., 1997)

13. R Philip, D Bevington, K Robinson, Data Reduction and Error Analysis for the Physical Sciences (McGraw-Hill, New York, 2003)

14. M Akcakaya, CH Muravchik, A Nehorai, Biologically inspired coupled antenna array for direction of arrival estimation. IEEE Trans. on Signal Processing 59(10), 4795-4808 (2011)

15. QY Yin, RW Newcomb, LH Zou, Estimating 2-D angle of arrival via two parallel linear array, in Proc. of IEEE International Conference on ASSP (IEEE, College Park, 1989), pp. 2803-2806

16. L Jun, L Guisheng, J Ming, M Qian, Multitarget detection and localization method for bistatic MIMO radar (Proceedings of IET International Radar Conference 2009, Guilin, China, 2009)

17. CP Mathews, MD Zoltowski, Eigenstructure techniques for 2-D angle estimation with uniform circular array. IEEE Trans. on Signal Processing 42(9), 2395-2407 (1994)

doi:10.1186/1687-6180-2013-81

Cite this article as: Li et al:: Multiple target three-dimensional coordinate estimation for bistatic MIMO radar with uniform linear receive array. EURASIP Journal on Advances in Signal Processing 2013 2013:81.

\section{Submit your manuscript to a SpringerOpen ${ }^{\circ}$ journal and benefit from:}

- Convenient online submission

- Rigorous peer review

- Immediate publication on acceptance

- Open access: articles freely available online

- High visibility within the field

- Retaining the copyright to your article

Submit your next manuscript at $\gg$ springeropen.com 